\title{
Spatial modeling of multicomponent pollution removal for liquid treatment under identification of mass transfer coefficient
}

\author{
Bomba A. ${ }^{1}$, Safonyk A. ${ }^{2}$, Voloshchuk V. ${ }^{3}$ \\ ${ }^{1}$ Rivne State Humanitarian University, \\ 12 Stepan Bandera Str., Rivne, 33000, Ukraine \\ ${ }^{2}$ Department of Automation, Electrical and Computer-Integrated Technologies, \\ National University of Water and Environmental Engineering, \\ 11 Soborna Str., Rivne, 33028, Ukraine \\ ${ }^{3}$ Department of Automation of Heat and Power Engineering Processes, \\ National Technical University of Ukraine "Igor Sikorsky Kyiv Polytechnic Institute", \\ 37 Prosp. Peremohy, Kyiv, 03056, Ukraine
}

(Received 2 October 2018)

\begin{abstract}
A generalized spatial mathematical model of the multicomponent pollutant removal for a liquid treatment is proposed. Under the assumption of domination of convective processes over diffusive ones, the model considers an inverse influence of the determining factor (pollution concentration in water and sludge) on the media characteristics (porosity, diffusion) and takes into account the specified additional condition (overridden condition) for estimation of the unknown mass transfer coefficient of a small value.

The algorithm for solving the corresponding nonlinear singularly perturbed inverse problem of the type "convection-diffusion-mass transfer" is developed. A computer experiment has been carried out based on this methodology.
\end{abstract}

Keywords: multicomponent pollutant, inverse problem, overdeterminatiom condition, asymptotic solution, identification, spatial modeling.

2000 MSC: 65E05, 65M25, 65M $32,68 \mathrm{U} 20,65 \mathrm{C} 20$

UDC: $519.63: 532.5$

DOI: $10.23939 / \mathrm{mmc} 2018.02 .108$

\section{Introduction}

In many areas of industry the difficult technological process of filtration is used by means of porous media. It is taken into account that liquid that is filtered through porous media contains some admixtures. These admixtures are distinguished by physical and chemical properties and can interact. The threats of ecocatastrophes are acquiring the special actuality through the insufficient cleaning of technological liquids in industry, for example, in energy and processing industries. To avoid such risks it is necessary to develop new methods that assist the intensification of mechanisms of water solution cleaning and increasing of work efficiency of water-purifying stations, creation of mathematical models of filtration processes through porous media. Analysis of the results presented in [1-11] indicates about complicated structure of mutual dependence of different factors which determine filtration through porous media and were not taken into account on conventional (phenomenological) models. The motivation for constructing the mathematical model of multicomponent pollutant removal for liquid treatment in spatial filter is the absence of "modeling mechanisms" which consider inverse influence of different kinds of the process and the media characteristics. The identification of unknown parameters is also of high priority. In $[12,13]$ a one-dimensional mathematical model of liquid treatment in filtering nozzle is developed which takes into account inverse influence of the process characteristics (sludge concentration) on filtering parameters. Some coefficients of the considered process were determined experimentally. The given work [13] is summarized on the space which corresponds to real filters. The mathematical model of multicomponent pollutant removal for liquid treatment in spatial filter taking into account 
unknown mass transfer coefficient with small value under assumption of domination of convective processes over diffusive ones. Solving appropriate inverse problem gives an opportunity to make calculation results very close to the experimental ones (compared with conventional, phenomenological models. It also estimates more exactly the effectiveness of impurity deposition in different technological water dispersion systems.

\section{Statement of the problem}

Consider a curvilinear parallelepiped (filter) $G_{z}=A B C D A_{*} B_{*} C_{*} D_{*}$, bounded by smooth orthogonal and equipotential surfaces between themselves at angular points and edges $A B B_{*} A_{*}=\left\{z: f_{1}(x, y, z)=\right.$ $0\}, C D C_{*} D_{*}=\left\{z: f_{2}(x, y, z)=0\right\}$, and also flow surfaces $A D D_{*} A_{*}=\left\{z: f_{3}(x, y, z)=0\right\}, B C C_{*} B_{*}=$ $\left\{z: f_{4}(x, y, z)=0\right\}, A B C D=\left\{z: f_{5}(x, y, z)=0\right\}, A_{*} B_{*} C_{*} D_{*}=\left\{z: f_{6}(x, y, z)=0\right\}$.

Assume [14] that the pollutants can transfer from one state to another (processes of adhesion and avulsion, sorption-desorption). The pollution concentrations influence the medium characteristics (porosity, filtration etc.) and are multicomponent $\left(C=C(x, y, z, t)=\left(C_{1}, \ldots, C_{m}\right)=\right.$ $\left.\left(C_{1}(x, y, z, t), \ldots, C_{m}(x, y, z, t)\right)\right), \quad$ where $C_{i}$ is the concentration of $i$-th impurity component $(i=\overline{1, m})$ in liquid filter medium. The corresponding process of filtration for the domain $G=C_{z} \times(0, \infty)$ is described by the following modeling problem $[9-11,14-17]$ :

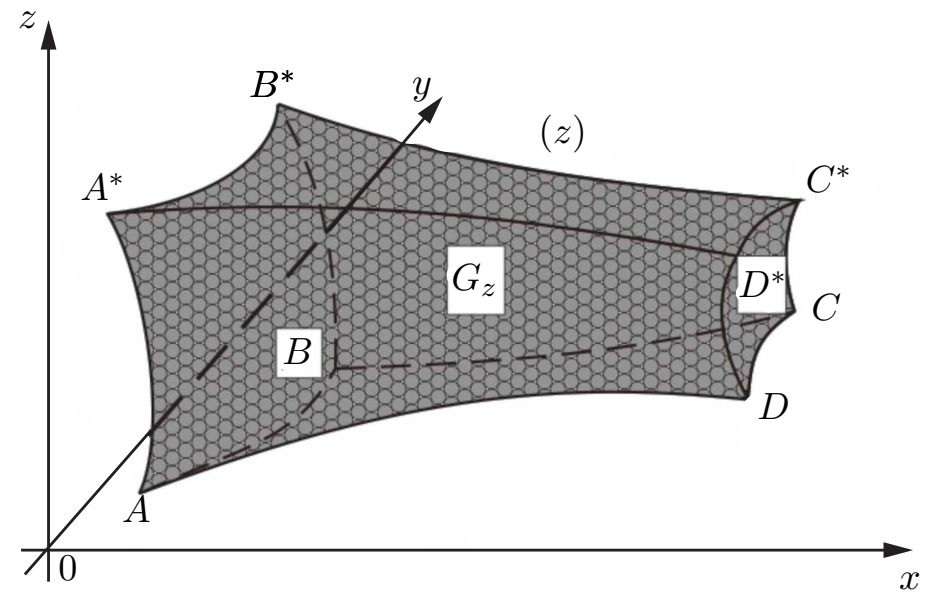

Fig. 1. Curvilinear parallelepiped (filter) $G_{z}$.

$$
\begin{gathered}
\left\{\begin{array}{c}
D_{i} \Delta C_{i}-\frac{\partial\left(\sigma(P) C_{i}\right)}{\partial t}-\boldsymbol{v} \cdot \nabla C_{i}-\varepsilon \sum_{\substack{l, g=1 \\
l \neq g}}^{m} k_{l, g} C_{l} C_{g}=\frac{\partial P}{\partial t}, \\
\frac{\partial P}{\partial t}=\left(\sum_{i=1}^{m} \beta_{i} C_{i}\right)-\varepsilon \alpha(t) P, \quad i=\overline{1, m}
\end{array}\right. \\
\left.C_{i}\right|_{A B B_{*} A_{*}}=C_{i, *}(M, t),\left.\quad \frac{\partial C_{i}}{\partial \boldsymbol{n}}\right|_{C D D_{*} C_{*}}=0,\left.\quad \frac{\partial C_{i}}{\partial \boldsymbol{n}}\right|_{A D D_{*} A_{*} \cup B C C_{*} B_{*} \cup A B C D \cup A_{*} B_{*} C_{*} D_{*}}=0, \\
C_{i}(x, y, z, 0)=C_{i, 0}^{0}(x, y, z), \quad P(x, y, z, 0)=P_{0}^{0}(x, y, z), \\
\boldsymbol{v}=\kappa(P) \nabla \varphi, \quad \nabla \cdot \boldsymbol{v}=0, \\
\left.\varphi\right|_{A B B_{*} A_{*}}=\varphi_{*},\left.\varphi\right|_{C D D_{* *}}=\varphi^{*}, \\
\left.\frac{\partial \varphi}{\partial \boldsymbol{n}}\right|_{A D D_{*} A_{*} \cup B C C_{*} B_{*} \cup A B C D \cup A_{*} B_{*} C_{*} D_{*}}=0, \\
\alpha(t) \iint_{G} P(\tilde{x}, \tilde{y}, \tilde{z}, t) d \tilde{x} d \tilde{y} d \tilde{z}=\mu(t),
\end{gathered}
$$

where $P(x, y, z, t)$ is the concentration of the sediment in the internal point $(x, y, z)$ of the domain $G$ (filter medium) at time $t ; \beta_{i}$ are the coefficients which characterize the amount of deposition per time unit; $\alpha(t)$ is unknown coefficient which expresses the amount of particles avulsed from the granular 
media filter; $\mu(t)$ is a function defining mass distribution of the sludge during time (is found experimentally [15]); (5) is overriding condition; $\sigma(P)$ is the porosity of medium $\left(\sigma(P)=\sigma_{0}-\varepsilon \sigma_{*} P(x, y, z, t)\right)$; $\boldsymbol{\nabla}$ is the Hamiltonian operator; $\Delta=\boldsymbol{\nabla} \cdot \boldsymbol{\nabla}$ is the Laplace operator; $D_{i}=d_{0 i} \varepsilon$ is the coefficient of diffusion of pollutants into the liquid; $\sigma_{*}, d_{0 i}, \varepsilon$ are solid parameters (characterize the corresponding soft parameter $\sigma(P)$ ), which are determined experimentally, $\varepsilon$ is the small parameter (it expresses advantages of some components of process over others, namely, desorptional components and phenomenon of component interaction of this process are small in comparison with other components); $C_{i}^{*}(M, t), C_{i, 0}^{0}(x, y, z)$ are smooth enough functions coherent between themselves on the edges of domain $G ; M$ is an arbitrary point of corresponding surface; $\varphi$ is the filtration potential $\left(0<\varphi_{*} \leqslant \varphi \leqslant \varphi^{*}<\infty\right) ; \boldsymbol{v}\left(v_{x}, v_{y}, v_{z}\right)$ is the vector of filtration rate $\left(|\boldsymbol{v}|>v_{*} \gg \varepsilon\right) ; \kappa=K(P)$ is the coefficient of filtration of corresponding porous medium $(K(P)$ is the given sufficiently smooth function; $\boldsymbol{n}$ the external normal to the corresponding surface. Introducing pair of the functions, $\psi=\psi(x, y, z), \eta=\eta(x, y, z)$ (spatially quasi-complex conjugated with the function $\varphi(x, y, z)$ ) such that $\kappa \cdot \operatorname{grad} \varphi=\operatorname{grad} \psi \times \operatorname{grad} \eta$ and substituting the boundary conditions: $\left.\psi\right|_{A D D_{*} A_{*}}=0,\left.\psi\right|_{B C C_{*} B_{*}}=Q_{*}$, $\left.\eta\right|_{A B C D}=0,\left.\eta\right|_{A_{*} D_{*} C_{*} B_{*}}=Q^{*}$, this problem is replaced by more general direct problem of finding spatial analogue of quasiconformal mapping the domain $G_{\mathbf{z}}$ on the corresponding domain of the complex quasi-potential $G_{w}=\left\{w=(\varphi, \psi, \eta): \varphi_{*} \leqslant \varphi \leqslant \varphi^{*}, 0<\psi<Q_{*}, 0<\eta<Q^{*}\right\}$, where $Q_{*}, Q^{*}$ are unknown parameters, $Q_{*} \cdot Q^{*}=Q=\int_{E F F_{*} E_{*}} \frac{\partial \varphi}{\partial s} d s$ amount of liquid that passes through some quasi-potentional surface $E F F_{*} E_{*}$ of domain $G_{\mathrm{z}}$ (total filtration rate). Assume that this problem on a spatial conformal mapping $G_{w} \mapsto G_{z}\left(G_{w}=\left\{w=(\varphi, \psi, \eta): \overline{\varphi_{*}}<\varphi<\overline{\varphi^{*}}, 0<\psi<Q_{*}, 0<\eta<Q^{*}\right\}\right.$ is corresponding $G_{z}$ domain of complex potential) at some average value of $\kappa$ was determined [8,13], in particular, dynamic net and the velocity field $\boldsymbol{v}$ are determined, filtration rate is calculated $Q=Q_{*} Q^{*}$. Then substituting variables $x=x(\varphi, \psi, \eta), y=y(\varphi, \psi, \eta), z=z(\varphi, \psi, \eta)$ in the system (1) and conditions (2), the corresponding problem for the domain $\left.G=C_{z} \times(0, \infty)\right)$ is formulated:

$$
\begin{gathered}
\left\{\begin{array}{l}
\varepsilon d_{0 i}\left(v^{2} \frac{\partial^{2} c_{i}}{\partial \varphi^{2}}+b_{1} \frac{\partial^{2} c_{i}}{\partial \psi^{2}}+b_{2} \frac{\partial^{2} c_{i}}{\partial \eta^{2}}+d_{1} \frac{\partial c_{i}}{\partial \psi}+d_{2} \frac{\partial c_{i}}{\partial \eta}\right) \\
-\frac{\partial\left(\sigma(\rho) c_{i}\right)}{\partial t}-v^{2} \frac{\partial c_{i}}{\partial \varphi}-\varepsilon \sum_{\substack{l, g=1 \\
l \neq g}}^{m} k_{l, g} c_{l} c_{g}=\frac{\partial \rho}{\partial t} \\
\frac{\partial \rho}{\partial t}=\sum_{i=1}^{m} \beta_{i} c_{i}-\varepsilon \alpha(t) \rho .
\end{array}\right. \\
c_{i}\left(\bar{\varphi}_{*}, \psi, \eta, t\right)=c_{i}^{*}(\psi, \eta, t), \quad c_{i, \varphi}\left(\bar{\varphi}^{*}, \psi, \eta, t\right)=0, \\
c_{i, \psi}(\varphi, 0, \eta, t)=c_{i, \psi}\left(\varphi, Q_{*}, \eta, t\right)=c_{i, \eta}(\varphi, \psi, 0, t)=c_{i, \eta}\left(\varphi, \psi, Q^{*}, t\right)=0, \\
\alpha(t) \iint_{G_{w}}^{0}(\varphi, \psi, \eta), \quad \rho(\varphi, \psi, \eta, 0)=\rho_{0}^{0}(\varphi, \psi, \eta),
\end{gathered}
$$

where (see, e.g. [15-17])

$$
\begin{aligned}
c_{i}= & c_{i}(\varphi, \psi, \eta, t)=C_{i}(x(\varphi, \psi, \eta), y(\varphi, \psi, \eta), z(\varphi, \psi, \eta), t), \\
\rho= & \rho(\varphi, \psi, \eta, t)=P(x(\varphi, \psi, \eta), y(\varphi, \psi, \eta), z(\varphi, \psi, \eta), t), \\
b_{1}= & b_{1}(\varphi, \psi, \eta)=(\nabla \psi)^{2}, \quad b_{2}=b_{2}(\varphi, \psi, \eta)=(\nabla \eta)^{2}, \\
d_{1}= & d_{1}(\varphi, \psi, \eta)=\Delta \psi, \quad d_{2}=d_{2}(\varphi, \psi, \eta)=\Delta \eta, \\
v^{2}(\varphi, \psi, \eta)= & v_{x}^{2}(x(\varphi, \psi, \eta), y(\varphi, \psi, \eta), z(\varphi, \psi, \eta))+v_{y}^{2}(x(\varphi, \psi, \eta), y(\varphi, \psi, \eta), z(\varphi, \psi, \eta)) \\
& +v_{z}^{2}(x(\varphi, \psi, \eta), y(\varphi, \psi, \eta), z(\varphi, \psi, \eta)) .
\end{aligned}
$$

Mathematical Modeling and Computing, Vol.5, No. 2, pp. 108-118 (2018) 
We used the methods of mathematical physics and hydrodynamics to build mathematical models of processes of cleaning liquids in which some components dominate the others - numerical-asymptotic methods; refinement methodology of the known classical models by going to the appropriate "perturbed" problems, thus preserving the classic form of laws that describe the processes of fluid flow in porous media, and the construction of their solutions without starting "first" supplement known "unperturbed" solution by various amendments.

\section{Asymptotic behavior of the solution}

The solution of the problem (6), (8) with accuracy $O\left(\varepsilon^{n}\right)$ asymptotic series [15-17]:

$$
\begin{gathered}
c_{i}=c_{i, 0}+\sum_{j=1}^{n} \varepsilon^{j} c_{i, j}+\sum_{j=0}^{n} \varepsilon^{j} \Pi_{i, j}+\sum_{j=0}^{n} \varepsilon^{j} \breve{\Pi}_{i, j}+\sum_{j=0}^{n+1} \varepsilon^{j / 2} \tilde{\Pi}_{i, j} \\
+\sum_{j=0}^{n+1} \varepsilon^{j / 2} \tilde{\tilde{\Pi}}_{i, j}+\sum_{j=0}^{n+1} \varepsilon^{j / 2} \hat{\Pi}_{i, j}+\sum_{j=0}^{n+1} \varepsilon^{j / 2} \hat{\hat{\Pi}}_{i, j}+R_{c, i}, \\
\rho=\rho_{0}+\sum_{j=1}^{n} \varepsilon^{j} \rho_{j}+\sum_{j=0}^{n} \varepsilon^{j} \bar{P}_{j}+\sum_{j=0}^{n} \varepsilon^{i} \breve{P}_{j}+\sum_{j=0}^{n+1} \varepsilon^{j / 2} \tilde{P}_{j} \\
+\sum_{j=0}^{n+1} \varepsilon^{j / 2} \tilde{\tilde{P}}_{j}+\sum_{j=0}^{n+1} \varepsilon^{j / 2} \hat{P}_{j}+\sum_{j=0}^{n+1} \varepsilon^{j / 2} \hat{\hat{P}}_{j}+R_{\rho}, \\
\alpha(t)=\alpha_{0}(t)+\sum_{j=1}^{k} \varepsilon^{j} \alpha_{j}(t)+R_{\alpha}(t, \varepsilon),
\end{gathered}
$$

where $R_{c, i}(\varphi, \psi, \eta, t, \varepsilon), \quad R_{\rho}(\varphi, \psi, \eta, t, \varepsilon), \quad R_{\alpha}(\varphi, \psi, \eta, t, \varepsilon)$ are remainder members, $c_{i, j}(\varphi, \psi, \eta, t)$, $\rho_{j}(\varphi, \psi, \eta, t), \alpha_{j}(t)$ are members of regular part of asymptotics $(i=\overline{1, m} ; j=\overline{0, n}) ; \Pi_{i, j}(\xi, \psi, \eta, t)$, $\bar{P}_{j}(\xi, \psi, \eta, t)$ are functions of the boundary type in the vicinity of the point $\varphi=\bar{\varphi}^{*}$ (corrections at the filter outlet) $(j=\overline{0,2}), \breve{\Pi}_{i, j}(\breve{\xi}, \psi, \eta, t), \breve{P}_{j}(\breve{\xi}, \psi, \eta, t)$ in the vicinity of the point $\phi=\bar{\varphi}^{*}$ (corrections at the filter intlet) $(j=\overline{0,2})$, and functions $\tilde{\Pi}_{i, j}(\varphi, \tilde{\psi}, \eta, t), \tilde{\tilde{\Pi}}_{i, j}(\varphi, \tilde{\tilde{\psi}}, \eta, t), \hat{\Pi}_{i, j}(\varphi, \psi, \tilde{\eta}, t), \hat{\Pi}_{i, j}(\varphi, \psi, \tilde{\tilde{\eta}}, t)$ and $\tilde{P}_{j}(\varphi, \tilde{\psi}, \eta, t), \tilde{\tilde{P}}_{j}(\varphi, \tilde{\tilde{\psi}}, \eta, t), \hat{P}_{j}(\varphi, \psi, \tilde{\tilde{\eta}}, t), \hat{\hat{P}}_{j}(\varphi, \psi, \tilde{\tilde{\eta}}, t)(j=\overline{0,3})$ in the vicinities of $\psi=0, \psi=Q^{*}$, $\eta=0, \eta=Q^{*}$ (vicinities of side walls of the filter), respectively; $\xi=\left(\varphi^{*}-\varphi\right) / \varepsilon, \breve{\xi}=\left(\varphi-\varphi_{*}\right) / \varepsilon$, $\tilde{\psi}=\psi / \sqrt{\varepsilon}, \tilde{\psi}=\left(Q_{*}-\psi\right) / \sqrt{\varepsilon}, \tilde{\eta}=\eta / \sqrt{\varepsilon}, \tilde{\tilde{\eta}}=\left(Q^{*}-\eta\right) / \sqrt{\varepsilon}$ are "stretches" of the corresponding variables. These functions are intended to take into account the boundary conditions on the side walls of the filter and allowed to calculate concentration in special zones in the case of a curvilinear form of the filter.

By the substitution (9)-(11) into (6)-(8) and fulfilling the standard procedure of equating coefficients at identical degrees $\varepsilon$, the following problems to solve $c_{i, j}(\varphi, \psi, \eta, t), \rho_{j}(\varphi, \psi, \eta, t)(j=\overline{0, n})$ are obtained:

$$
\left\{\begin{array}{l}
\sigma_{0} \frac{\partial c_{i, 0}}{\partial t}+v^{2} \frac{\partial c_{i, 0}}{\partial \varphi}+\sum_{i=1}^{m} \beta_{i} c_{i, 0}=0, \quad \frac{\partial \rho_{0}}{\partial t}=\sum_{i=1}^{m} \beta_{i} c_{i, 0} \\
c_{i, 0}(\varphi, \psi, \eta, 0)=c_{i, 0}^{0}, \quad c_{i, 0}\left(\varphi_{*}, \psi, \eta, t\right)=c_{i *}(\psi, \eta, t) \\
\rho_{0}(\varphi, \psi, \eta, 0)=\rho_{0}^{0} ; \\
\alpha_{0}(t) \iiint_{G_{w}} \rho_{0}(\tilde{\varphi}, \tilde{\psi}, \tilde{\eta}, t) d \tilde{\varphi} d \tilde{\psi} d \tilde{\eta}=\mu(t)
\end{array}\right.
$$

Mathematical Modeling and Computing, Vol. 5, No. 2, pp. 108-118 (2018) 


$$
\left\{\begin{array}{l}
\sigma_{*} \rho_{j-1} \frac{\partial c_{i, j}}{\partial t}-v^{2} \frac{\partial c_{i, j}}{\partial \varphi}-\sum_{\substack{l, g=1 \\
l \neq g}}^{m} k_{l, g} c_{l, j-1} c_{g, j-1}=U_{i, j} \\
\frac{\partial \rho_{j}}{\partial t}=\sum_{i=1}^{m} \beta_{i} c_{i, j}-\sum_{\substack{k=1 \\
j}} \alpha_{j-k}(t) \rho_{k-1}, \\
c_{i, j}(\varphi, \psi, \eta, 0)=0, \quad c_{i, j}\left(\varphi_{*}, \psi, \eta, t\right)=0, \quad \rho_{j}(\varphi, \psi, \eta, 0)=0
\end{array}\right.
$$

$\alpha_{0}(t) \iiint_{G_{w}} \rho_{0}(\tilde{\varphi}, \tilde{\psi}, \tilde{\eta}, t) d \tilde{\varphi} d \tilde{\psi} d \tilde{\eta}+\alpha_{1}(t) \iiint_{G_{w}} \rho_{j-1}(\tilde{\varphi}, \tilde{\psi}, \tilde{\eta}, t) d \tilde{\varphi} d \tilde{\psi} d \tilde{\eta}+\ldots+$

$$
+\alpha_{j}(t) \iiint_{G_{w}} \rho_{0}(\tilde{\varphi}, \tilde{\psi}, \tilde{\eta}, t) d \tilde{\varphi} d \tilde{\psi} d \tilde{\eta}=0
$$

Solving provides:

$$
\begin{gathered}
c_{i, 0}=\left\{\begin{array}{l}
c_{i, *}(\psi, \eta, t-f) \exp \left[-\beta_{i} \int_{\varphi_{*}}^{\varphi} \frac{d \tilde{\varphi}}{v^{2}(\tilde{\varphi}, \psi, \eta)}\right], \quad t \geqslant f, \\
c_{i, 0}^{0}\left(f^{-1}(f-t, \psi, \eta), \psi, \eta\right) \exp \left[-\frac{\beta_{i} t}{\sigma_{0}}\right], \quad t<f,
\end{array}\right. \\
\rho_{0}=\int_{0}^{t}\left(\sum_{i=1}^{m} \beta_{i} c_{i, 0}\right) d \tilde{t}+\rho_{0}^{0}, \quad \alpha_{0}(t)=\frac{\mu(t)}{\iiint_{G_{w}} \rho_{0}(\tilde{\varphi}, \tilde{\psi}, \tilde{\eta}, t) d \tilde{\varphi} d \tilde{\psi} d \tilde{\eta}}, \\
c_{i, j}=\left\{\begin{array}{l}
e^{-\lambda_{1}} \int_{\varphi_{0}}^{\varphi} \frac{U_{i, j}(s, \psi, \eta, f(s, \psi, \eta)-f+t)}{v^{2}(s, \psi, \eta)} e^{\lambda_{2}(s, \psi, \eta, t)} d s, \quad t \geqslant f, \\
-\frac{e^{-\lambda_{1}}}{\sigma_{*}} \int_{0}^{t} \frac{U_{i, j}\left(f^{-1}(s+f-t, \psi, \eta), \psi, \eta, s\right)}{\rho_{j-1}\left(f^{-1}(s+f-t, \psi, \eta), \psi, \eta\right)} e^{\lambda_{2}(\varphi, \psi, \eta, s)} d s, \quad t<f, \\
\rho_{j}=\int_{0}^{t}\left(\sum_{i=1}^{m} \beta_{i} c_{i, j}-\sum_{k=1}^{j} \alpha_{j-k}(t) \rho_{k-1}\right) d \tilde{t}, \quad \alpha_{j}(t)=\frac{\sum_{k=1}^{j} \alpha_{j-k}(t) \iiint_{G_{w}} \rho_{j}(\tilde{\varphi}, \tilde{\psi}, \tilde{\eta}, t) d \tilde{\varphi} d \tilde{\psi} d \tilde{\eta}}{\iiint_{G_{w}} \rho_{0}(\tilde{\varphi}, \tilde{\psi}, \tilde{\eta}, t) d \tilde{\varphi} d \tilde{\psi} d \tilde{\eta}}
\end{array}\right.
\end{gathered}
$$

where

$$
\begin{aligned}
& U_{i, j}(\varphi, \psi, \eta, t)=d_{0 i}\left(v^{2} \frac{\partial^{2} c_{i, j}}{\partial \varphi^{2}}+b_{1} \frac{\partial^{2} c_{i, j}}{\partial \psi^{2}}+b_{2} \frac{\partial^{2} c_{i, j}}{\partial \eta^{2}}+d_{1} \frac{\partial c_{i, j}}{\partial \psi}+d_{2} \frac{\partial c_{i, j}}{\partial \eta}\right)+\alpha_{j-1}(t) \rho_{j-1} \\
& -\sum_{\substack{l, g=1 \\
l \neq g}}^{m} k_{l, g} c_{l, j-1} c_{g, j-1}-\sum_{i=1}^{m} \beta_{i} c_{i, j},(j=\overline{2, n}), \\
& \lambda_{1}(\varphi, \psi, \eta, t)=-\beta_{i} \int_{\varphi_{0}}^{\varphi} \frac{\rho_{j-1}(s, \psi, \eta, f(\tilde{\varphi}, \psi, \eta)+t-f) c_{i, j}(s, \psi, \eta, f(\tilde{\varphi}, \psi, \eta)+t-f)}{v^{2}(s, \psi, \eta)} d s, \\
& \lambda_{2}(\varphi, \psi, \eta, t)=-\beta_{i} \int_{0}^{t} \frac{\rho_{j-1}\left(f^{-1}(\tilde{s}+f(\varphi, \psi, \eta)-t, \psi, \eta), \psi, \eta, \tilde{s}\right) c_{i, j}\left(f^{-1}(\tilde{s}+f(\varphi, \psi, \eta)-t, \psi, \eta), \psi, \eta, \tilde{s}\right)}{\sigma\left(f^{-1}(\tilde{t}+f(\varphi, \psi, \eta)-t, \psi, \eta), \psi, \eta\right)} d \tilde{s},
\end{aligned}
$$

$f(\varphi, \bar{\psi}, \bar{\eta})=\int_{\varphi_{0}}^{\varphi} \frac{d s}{v^{2}(s, \psi, \bar{\eta})}$ is the time transit made by of the corresponding particle from the point $\left(x\left(\varphi_{*}, \bar{\psi}, \bar{\eta}\right), y\left(\varphi_{*}, \bar{\psi}, \bar{\eta}\right), z\left(\varphi_{*}, \bar{\psi}, \bar{\eta}\right)\right) \in A B B_{*} A_{*}$ to the point $(x(\varphi, \bar{\psi}, \bar{\eta}), y(\varphi, \bar{\psi}, \bar{\eta}), z(\varphi, \bar{\psi}, \bar{\eta})) \in G_{\mathrm{z}}$ along the corresponding flow line (as cross section of two surfaces $\psi(x, y, z)=\bar{\psi}, 0 \leqslant \bar{\psi} \leqslant Q_{*}$, $\left.\eta(x, y, z)=\bar{\eta}, 0 \leqslant \bar{\eta} \leqslant Q^{*}\right) f^{-1}$ is the inverse of a function $f$ with respect to $\varphi$ (it should be noted that such function exists because $v^{2}(\phi, \psi, \eta)$ is continuously differentiable, limited and positive. Functions $\Pi_{i, j}(\xi, \psi, \eta, t), \bar{P}_{j}(\xi, \psi, \eta, t),(i=0,1) \breve{\Pi}_{i, j}(\breve{\xi}, \psi, \eta, t), \breve{P}_{j}(\breve{\xi}, \psi, \eta, t), \tilde{\Pi}_{i, j}(\varphi, \tilde{\mu}, \eta, t), \tilde{P}_{i, j}(\varphi, \tilde{\mu}, \eta, t)$, $\tilde{\tilde{\Pi}}_{i, j}(\varphi, \tilde{\tilde{\psi}}, \eta, t), \hat{\Pi}_{i, j}(\varphi, \psi, \tilde{\eta}, t), \hat{\hat{\Pi}}_{i, j}(\varphi, \psi, \tilde{\tilde{\eta}}, t)$ and $\tilde{\tilde{P}}_{j}(\varphi, \tilde{\tilde{\psi}}, \eta, t), \hat{P}_{j}(\varphi, \psi, \tilde{\eta}, t), \hat{\hat{P}}_{j}(\varphi, \psi, \tilde{\eta}, t)$ are determined in accordance with [8]. The estimation of remainder members is made in accordance with [14]. Taking 3-4 members of the asymptotic series (9)-(11) is sufficient for receiving approximate solutions with precision of 4 digits within calculation time of the filtering cycle [18]. 


\section{Results of numerical calculations}

Here are the results of numerical experiment. Here $L=1 \mathrm{~m}, \beta_{i}=0.3 \mathrm{~s}^{-1}, \alpha_{0}=0.0056 \mathrm{~s}^{-1}, \sigma_{0}=0.5$, $\varepsilon=0.001 ; k=1 ; c_{1,0}^{0}(\varphi, \psi, \eta)=0.02 \exp \left(-\varphi^{2}\right), c_{2,0}^{0}(\varphi, \psi, \eta)=0.015 \exp \left(-\varphi^{2}\right), c_{1}^{*}(\psi, \eta, t)=0.02$, $c_{2}^{*}(\psi, \eta, t)=0.015, \rho_{0}^{0}(\varphi, \psi, \eta)=0$. As a result of the interpolation of the experimental data [8] the distribution of mass of sludge $\mu(t)$ in time is obtained (see Fig. $2 a$ ). Time dependence of the respective mass transfer coefficient $\alpha(t)$ is shown in the (see Fig. $2 b$ ). The growth of mass transfer coefficient in time is due to the fact that for the experimentally obtained value $\mu(t)$ during the particles sedimentation the granules of the porous media are saturated with particles and under hydraulic pressure probability of avulsion of particles from the granules is increased to time $\tau$ of the effective filtering.
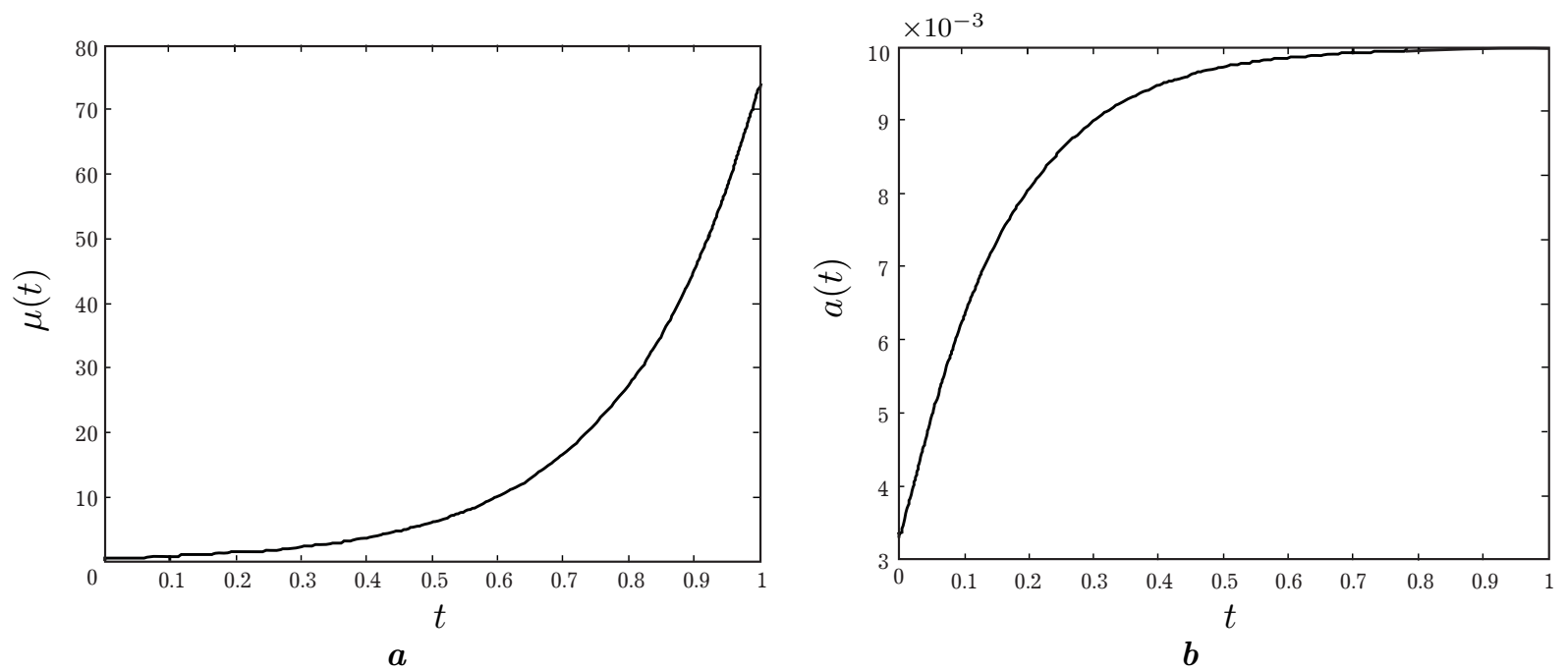

Fig. 2. The distribution of mass of sludge $\mu(t)(\boldsymbol{a})$ and mass transfer coefficient $\alpha(t)(\boldsymbol{b})$ in time.

Fig. 3 shows the results of calculating the concentration of pollution, obtained experimentally and according to the built model for $c_{1}=0.02, c_{2}=0.015$. Analysis of these results suggests the consistency of experimental and calculated data.
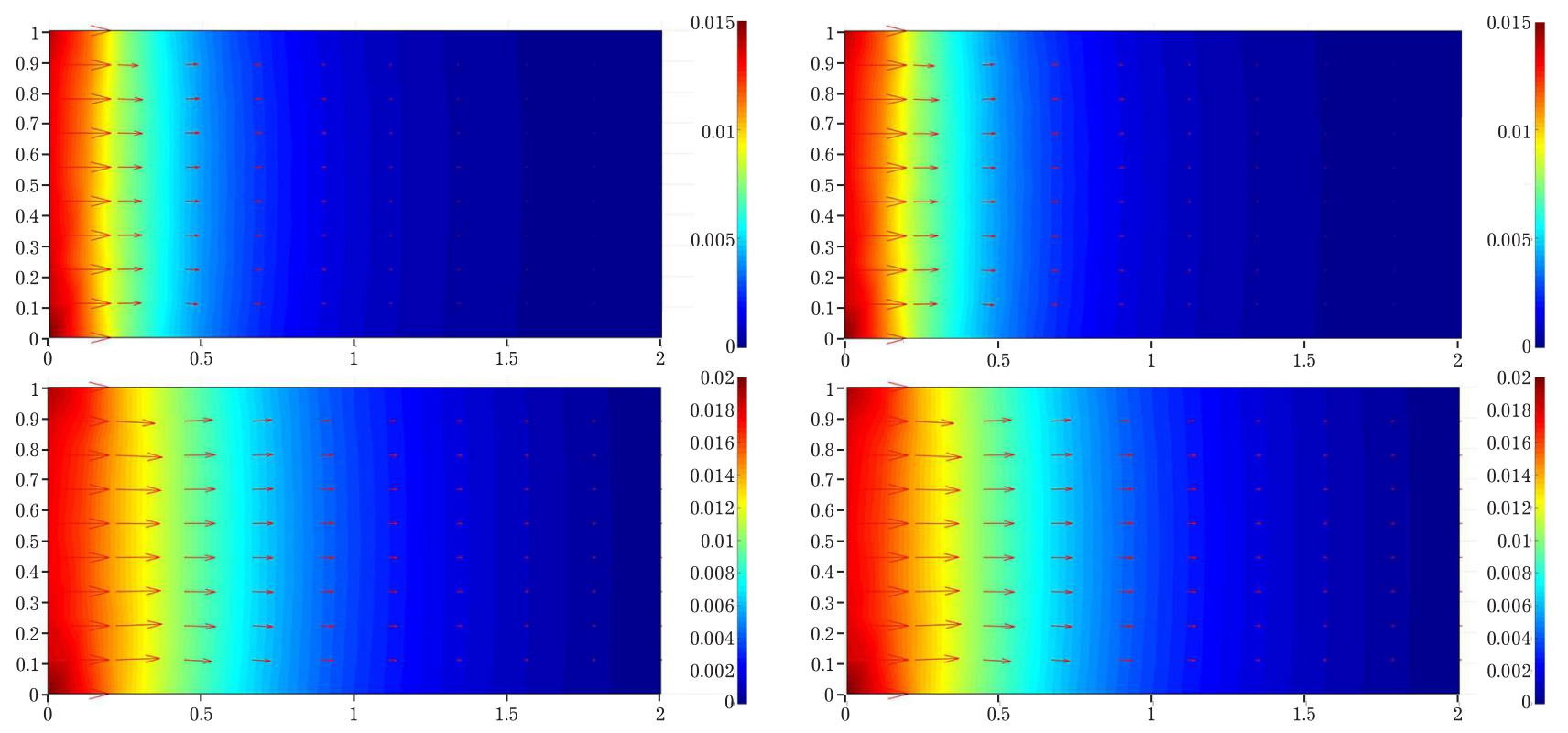

Fig. 3. Results of calculating the concentration of pollution, obtained experimentally and according to the built model for $c_{1}=0.02, c_{2}=0.015$. 

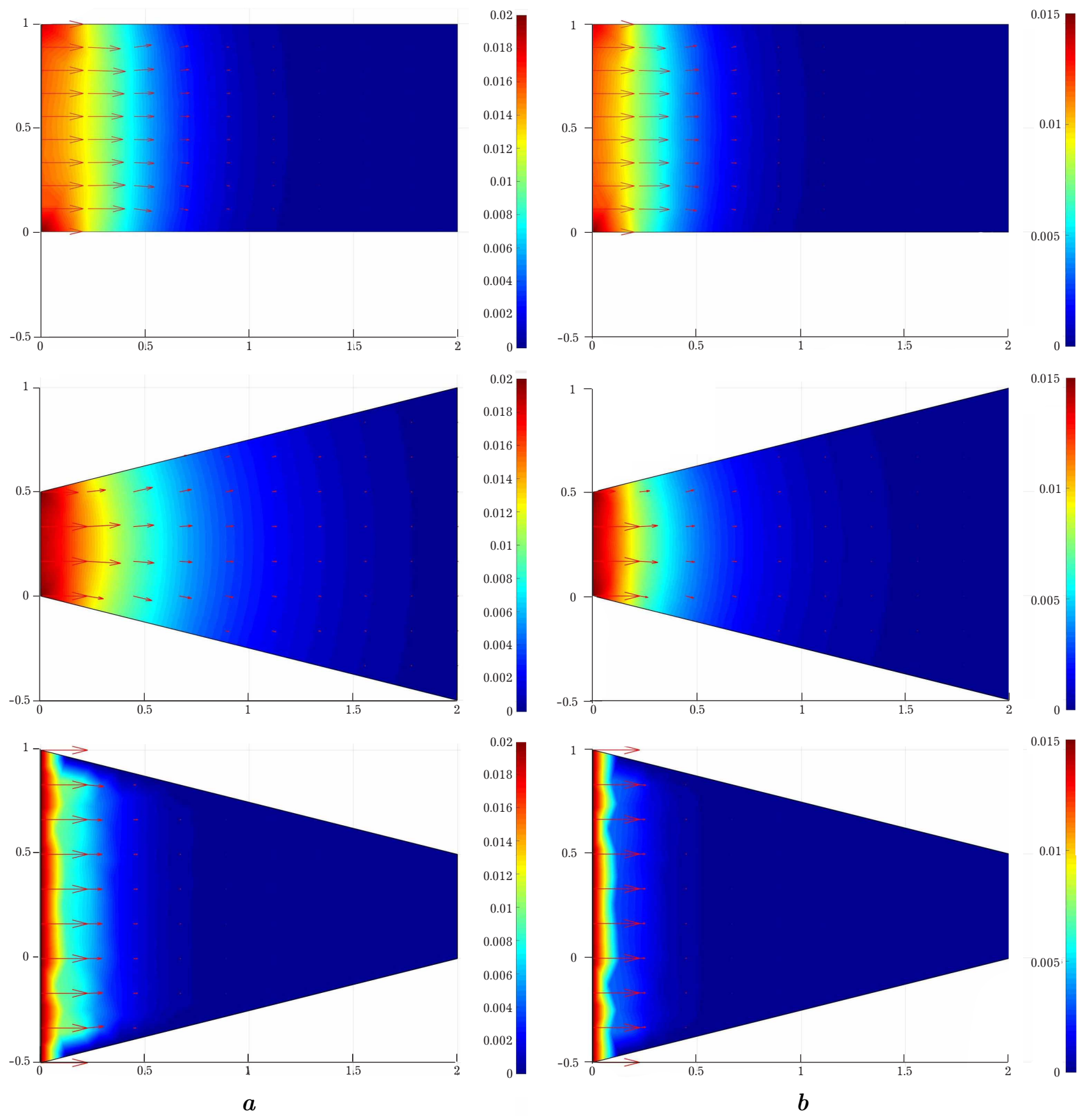

Fig. 4. Distribution of pollution concentration along the filter after 5 hours: $(\boldsymbol{a})-c_{1}=0.02 ;(\boldsymbol{b})-c_{2}=0.015$.

Figs. 4-6 show the results of computer simulation of the purification process by different shapes filters. The obtained results allowed to estimate the efficiency of the purification depending on the geometric characteristics of the calculated area. 

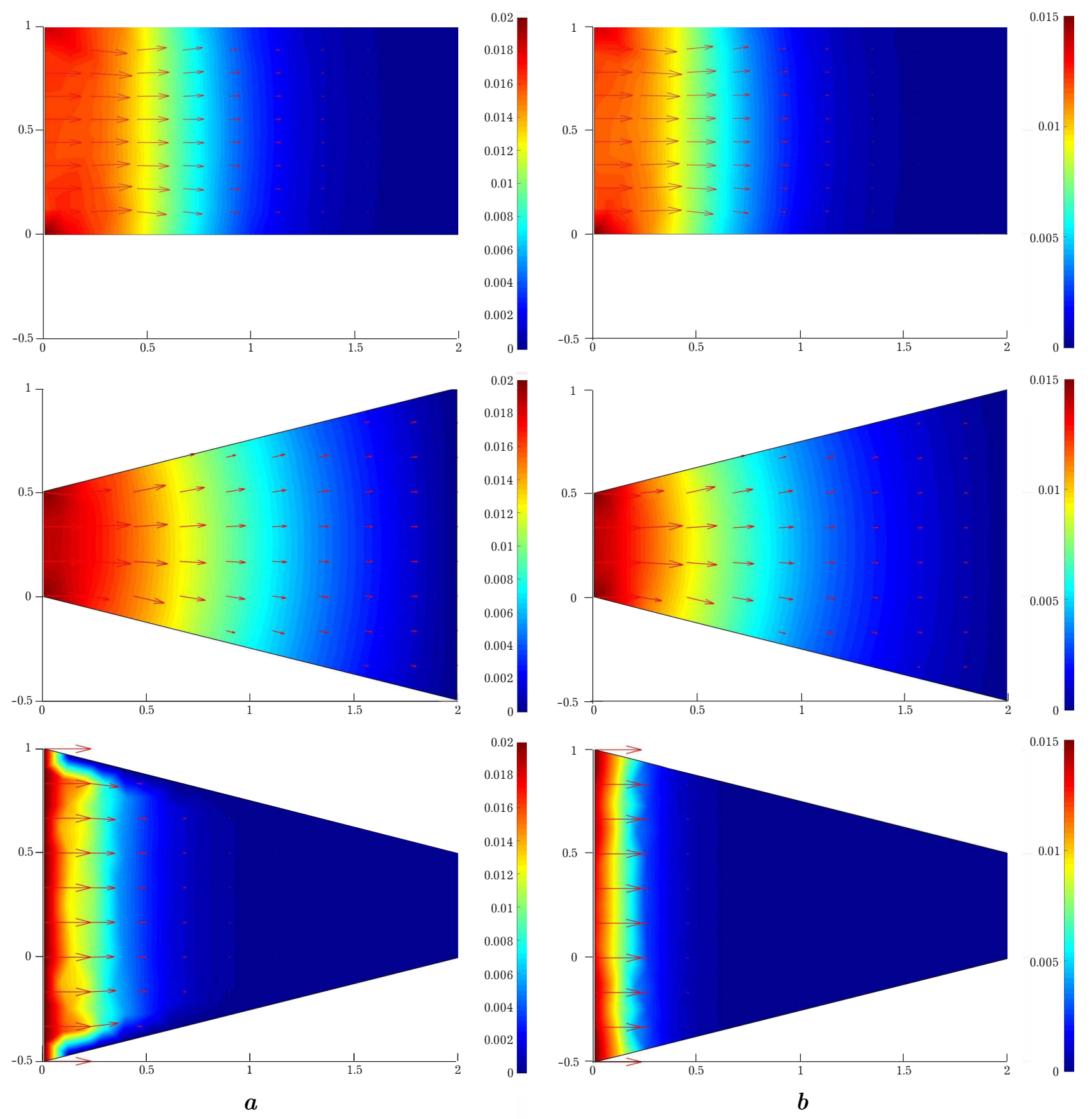

Fig. 5. Distribution of pollution concentration along the filter after 10 hours: $(\boldsymbol{a})-c_{1}=0.02 ;(\boldsymbol{b})-c_{2}=0.015$. 

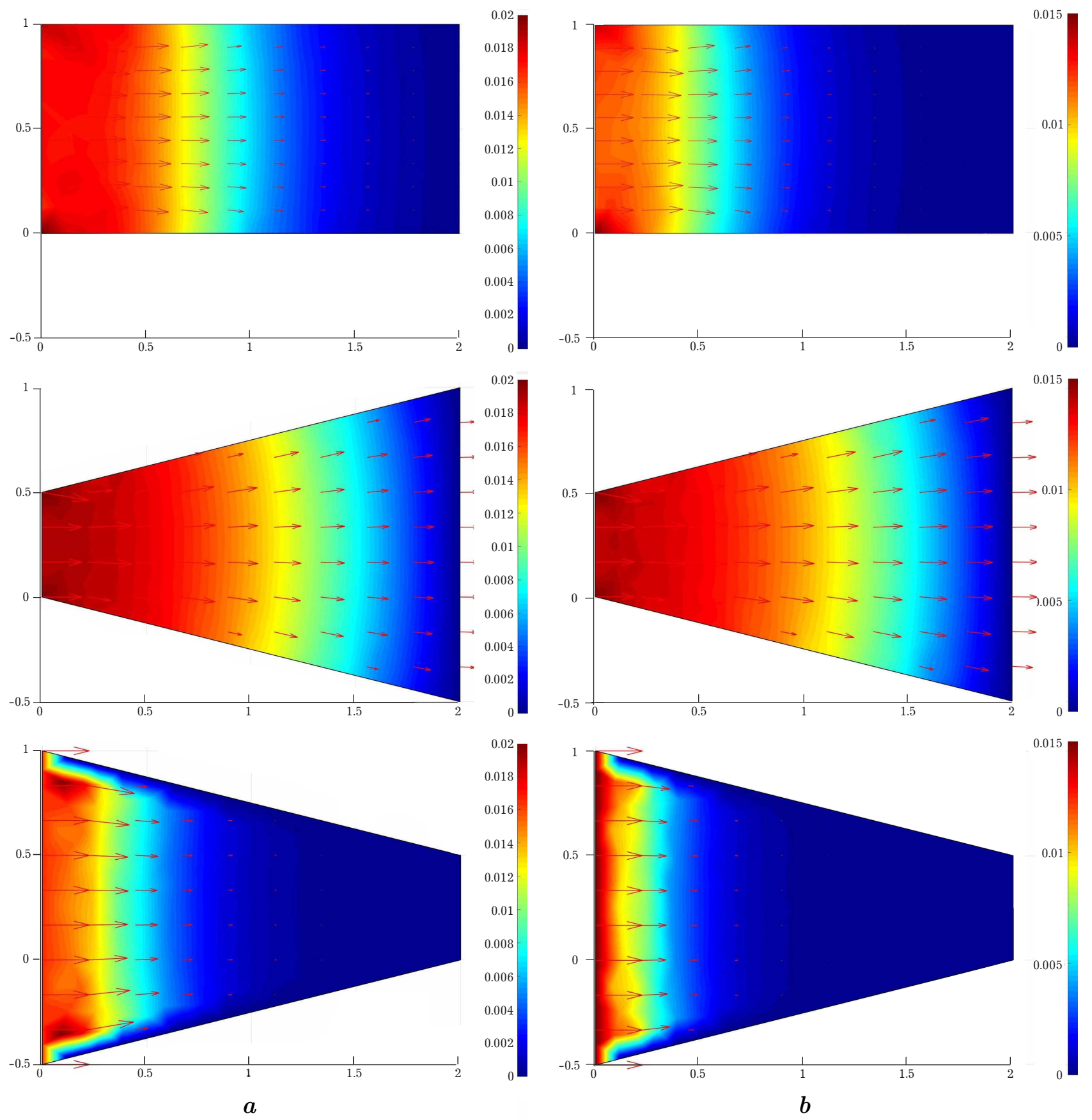

Fig. 6. Distribution of pollution concentration along the filter after 15 hours: $(\boldsymbol{a})-c_{1}=0.02 ;(\boldsymbol{b})-c_{2}=0.015$.

After analyzing the results of computer experiment (see Figs. 4-6), we come to the following conclusions: specifying the shape of a filter plays a significant role in the filtration process because it can lead both to the increase (decrease) of the filtering parameters and to the increase of the productivity of its work in general.

\section{Conclusions}

A generalized spatial mathematical model of multicomponent pollutant removal for liquid treatment is proposed. Under the assumption of domination of convective processes over diffusive ones, the model considers an inverse influence of determining factor (pollution concentration in liquid and sludge) on the media characteristics (porosity, diffusion) and takes into account the specified additional condition 
(overridden condition) for estimation of the unknown mass transfer coefficient of a small value. The solving of the corresponding inverse problem is presented which gives us an opportunity to make calculation results very close to the experimental ones (compared with conventional, phenomenological models [8]) and estimate more exactly the effectiveness of impurity deposition in different technological water dispersion systems. The analysis of the investigations results shows that the filter form plays a significant role in the filtering because it can lead to an increase (decrease) of filtering parameters and to the increase the productivity.

[1] Berg C. Permeability description by characteristic length, tortuosity, constriction and porosity. Transport in Porous Media. 103 (3), 381-409 (2014).

[2] Calo V., Iliev O., Lakdawala Z., Leonard K., Printsypar G. Pore-scale modeling and simulation of flow, transport, and adsorptive or osmotic effects in membranes: The influence of membrane microstructure. International journal of advances in engineering sciences and applied mathematics. 7 (1), 2-13 (2015).

[3] Chetti A., Benamar A., Abdelkrim H. Modeling of Particle Migration in Porous Media: Application to Soil Suffusion. Transport in Porous Media. 113 (3), 591-606 (2016).

[4] Civa F. Modified formulations of particle deposition and removal kinetics in saturated porous media. Transport in Porous Media. 111 (2), 381-410 (2016).

[5] Gravelle A., Peysson Y., Tabary R., Egermann P. Experimental investigation and modelling of colloidal release in porous media. Transport in Porous Media. 88 (3), 441-459 (2011).

[6] Husseinab M., Lesnica D., Ivanchov M., Snitkod H. Multiple time-dependent coefficient identification thermal problems with a free boundary. Appl. Num. Math. 99, 24-50 (2016).

[7] Mittal R., Jain R. Numerical solutions of nonlinear Fisher's reaction-diffusion equation with modified cubic B-spline collocation method. Math. Sci. 7 (1), 12 (2013).

[8] Peng Ch., Xu G., Wu W., Yu H., Wangc Ch. Multiphase SPH modeling of free surface flow in porous media with variable porosity. Computers and Geotechnics. 81, 239-248 (2017).

[9] Shi M., Printsypar G., Iliev O., Calo V., Amy G., Nunes S. Water flow prediction based on 3D membrane morphology simulation. J. Membr. Sci. 487, 19-31 (2015).

[10] Orlov V., Martynov S., Kunitskiy S. Energy saving in water treatment technologies with polystyrene foam filters. Journal of Water and Land Development. 31 (X-XII), 119-122 (2016).

[11] Orlov V., Martynov S., Kunitskiy S. Water defferrization in polystyrene foam filters with sediment layer. LAP LAMBERT Academic Publishing, Saarbrucken. 94 p. (2016).

[12] Bomba A., Safonyk A., Fursachik E. Identification of mass transfer distribution factor and its account for magnetic filtration process modeling. Journal of Automation and Information Sciences. 45 (4), 16-22 (2013).

[13] Orlov V., Safonyk A., MartynovS. Simulation the process of iron removal the underground water by polystyrene foam filters. International Journal of Pure and Applied Mathematics. 109 (4), 881-888 (2016).

[14] Safonyk A. Modelling the filtration processes of liquids from multicomponent contamination in the conditions of authentication of mass transfer coefficient. Int. J. Math. Models and Methods in Appl. Sciences. 9, 189-192 (2015).

[15] Safonyk A. Modelling of biological purification process taking into account the temperature mode. Int. J. Math. Models and Methods in Appl. Sciences. 12, 68 (2018).

[16] Bomba A., Klymiuk Yu., Prysiazhniuk I., Prysiazhniuk O., Safonyk A. Mathematical modeling of wastewater treatment from multicomponent pollution by using microporous particles. AIP Conference Proceedings. 1773 (1), 040003 (2016).

[17] Bomba A., Safonik A. Mathematical simulation of the process of aerobic treatment of wastewater under conditions of diffusion and mass transfer perturbations. Journal of Engineering Physics and Thermophysics. 91 (2), 318-323 (2018).

[18] Safonyk A., Martynov S., Kunytsky S., Pinchuk O. Mathematical modelling of regeneration the filtering media bed of granular filters. Advances in Modelling and Analysis C. 73 (2). $72-78$ (2018). 


\title{
Просторове моделювання процесу очищення рідини від багатокомпонентного забруднення за умов ідентифікації масообмінного коефіцієнта
}

\author{
Бомба А. ${ }^{1}$, Сафоник А. ${ }^{2}$, Волощук В. ${ }^{3}$ \\ ${ }^{1}$ Рівненсъкий державний гуманітарний університет, \\ вул. Степана Бандери, 12, Рівне, 33000, Україна \\ ${ }^{2}$ Національний уніваерситет водного господарства та природокористування, \\ вул. Соборна, 11 33028, Рівне, Україна \\ ${ }^{3}$ Національний технічний університет України \\ "Київский політехнічний інститут імені Ігоря Сікорсъкого", \\ nросп. Перемоги, 37, Київ, 03056, Україна
}

\begin{abstract}
Запропоновано просторове узагальнення математичної моделі очищення рідини від багатокомпонентного забруднення, яка, за припущення про домінування конвективних складових цього процесу над дифузійними, враховує зворотний вплив визначальних факторів (концентрації забруднення рідини та осаду) на характеристики середовища (коефіцієнт пористості, дифузії), і містить спеціально задану додаткову умову (умову перевизначення) для знаходження невідомого малого масообмінного коефіцієнта. Побудовано алгоритм розв'язування відповідної нелінійної оберненої сингулярно збуреної задачі типу "конвекція-дифузія-масообмін”. На цій основі проведено комп'ютерний експеримент.
\end{abstract}

Ключові слова: багатокомпонентне забруднення, обернена задача, умова перевизначення, асимптотичний розв'язок, ідентифікація, просторова модель.

2000 MSC: 65E05, 65M25, 65M32, 68U20, 65C20

УдК: $519.63: 532.5$ 